ARTICLE

https://doi.org/10.1038/s41467-020-14615-3

\title{
De novo strategy with engineering anti-Kasha/ Kasha fluorophores enables reliable ratiometric quantification of biomolecules
}

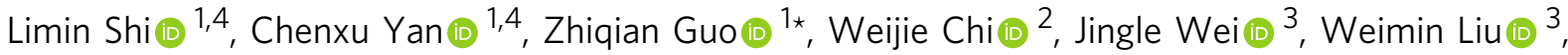 \\ Xiaogang Liu (i) ${ }^{2 \star}$, He Tian (D) ${ }^{1} \&$ Wei-Hong Zhu (1) ${ }^{1 \star}$
}

Fluorescence-based technologies have revolutionized in vivo monitoring of biomolecules. However, significant technical hurdles in both probe chemistry and complex cellular environments have limited the accuracy of quantifying these biomolecules. Herein, we report a generalizable engineering strategy for dual-emission anti-Kasha-active fluorophores, which combine an integrated fluorescein with chromene (IFC) building block with donor- $\pi$-acceptor structural modification. These fluorophores exhibit an invariant near-infrared Kasha emission from the $S_{1}$ state, while their anti-Kasha emission from the $S_{2}$ state at around $520 \mathrm{~nm}$ can be finely regulated via a spirolactone open/closed switch. We introduce bio-recognition moieties to IFC structures, and demonstrate ratiometric quantification of cysteine and glutathione in living cells and animals, using the ratio $\left(S_{2} / S_{1}\right)$ with the $S_{1}$ emission as a reliable internal reference signal. This de novo strategy of tuning anti-Kasha-active properties expands the in vivo ratiometric quantification toolbox for highly accurate analysis in both basic life science research and clinical applications.

\footnotetext{
${ }^{1}$ Key Laboratory for Advanced Materials and Joint International Research Laboratory of Precision Chemistry and Molecular Engineering, Feringa Nobel Prize Scientist Joint Research Center, Institute of Fine Chemicals, School of Chemistry and Molecular Engineering, East China University of Science and Technology, Shanghai 200237, China. ${ }^{2}$ Science and Math Cluster, Singapore University of Technology and Design, 8 Somapah Road, Singapore 487372, Singapore.

${ }^{3}$ School of Physical Science and Technology, ShanghaiTech University, Shanghai 201210, China. ${ }^{4}$ These authors contributed equally: Limin Shi, Chenxu Yan.

*email: guozq@ecust.edu.cn; xiaogang_liu@sutd.edu.sg; whzhu@ecust.edu.cn
} 
F luorescence technologies have revolutionized the way we study life science and conduct medical diagnostics ${ }^{1-6}$. It is now a routine process to directly visualize biomolecules in both fixed and live cells using fluorescence microscopy, which affords high spatial and temporal resolution down to even the single molecule level in a fraction of a second $d^{7-10}$. In parallel with the rapid evolution of fluorescent imaging techniques, increased attention is now focused on the accurate quantitative measurement of biomolecules via fluorescent signals ${ }^{11-14}$. However, in a highly heterogeneous and dynamic biological milieu, obtaining quantitative information of biomolecules remains a significant challenge ${ }^{15}$ : The emission intensities of fluorescent probes are affected by both the target biomolecules and other extraneous factors such as probe concentrations, excitation power, and cellular context.

To eliminate the impact of extraneous factors, one solution is to employ two fluorophores with equal or correlated concentrations to ratiometrically quantify biomolecules. To this end, several techniques have been developed, such as Förster resonance energy transfer (FRET) and unimolecular based ratiometric probes (Fig. 1a, b). The most prominent technology behind these methods is the FRET mechanism, which uses a single excitation source but acquires two emission bands from a set of paired reporter fluorophores (Fig. 1a) ${ }^{16}$. Monitoring the ratiometric changes in the emission intensity of these two paired fluorophores enables the quantification of a target analyte ${ }^{17}$. Despite its undeniably huge impact in research, many issues still complicate FRET measurements, some of which can cause misleading or even render meaningless results, for example inevitable excitation/emission cross-talk ${ }^{18}$, conformational and/or microenvironmental changes between the energy donor and acceptor fluorophores ${ }^{19}$, and different photobleaching efficiencies of the two fluorophores. Thus, FRET requires sophisticated calibrations to generate reliable signals.

It has been established that the preferential ratiometric patterns of a unimolecular fluorophore can overcome some inevitable side effects between donor and acceptor fluorophores in FRET-based methods ${ }^{20-23}$. Unimolecular ratiometric probes can react with the analyte to form a new fluorophore. The emission intensity ratios between the reactant and product fluorophores-which are typically excited at two distinct wavelengths-can enable quantification of the analyte (Fig. 1b). Nevertheless, in dynamic cellular environment, different photobleaching rates and concentration-dependent diffusion properties of the reactant and the product fluorophores have limited the widespread applications of such unimolecular probes. Moreover, it is challenging for unimolecular probes to establish a suitable and reliable internal intensity reference to enable accurate quantitative measurements 24,25 .

One effective approach to address these challenges would be to develop a single-type of probe with dual-emission signals: one serving as the internal intensity reference and the other for representing interactions with the target analyte (Fig. 1c). Unfortunately, such probes are often deemed impossible in that they defy Kasha's rule. As an important principle in photochemistry to most fluorophores, Kasha's rule states that fluorophores can only generate one emission band from the lowestenergy excited singlet state $\left(S_{1}\right)$, irrespective of the excitation wavelength ${ }^{26}$. In contrast, anti-Kasha dyes remain exceedingly rare $27-32$, and most of them emit in the short wavelength region and are thus not suitable for biological applications ${ }^{33}$. Moreover, to the best of our knowledge, the modulation of anti-Kasha emission for ratiometrically detecting bioanalytes has yet to be demonstrated.

Herein, we report a general strategy to construct activatable anti-Kasha dual-emission fluorophores based on a discovered building block, which we coin as integrated fluorescein with chromene (IFC) chromophores, wherein a spirolactone open/ closed switch can finely regulate anti-Kasha-active behavior. Using the designed probes such as dicyanomethylene- $4 \mathrm{H}$-pyran (DCM)-IFC, we were able to conduct in vivo measurement and quantification of biomolecules at the physiological $\mathrm{pH}$ condition. The near-infrared (NIR) Kasha emission signal $(700 \mathrm{~nm})$ from $\mathrm{S}_{1}$ state of DCM-IFC remains nearly constant, while its anti-Kasha emission signal from $\mathrm{S}_{2}$ state increases linearly with analyte concentrations via a spirolactone open/closed switch. The ratiometric bioimaging based on the single fluorophore allows us to accurately perform quantification of various biomolecules in living cells and a mice model. This de novo strategy for activatable anti-Kasha fluorophores would greatly facilitate the advancement of quantitative fluorescence imaging.

\section{Results}

Revealing the anti-Kasha properties of open-form DCM-IFC. Our group has been engaged in a long-term research project investigating the use of high-performance donor- $\pi$-acceptor (D- $\pi$-A) fluorescent dyes ${ }^{34,35}$, such as DCM, QM, and BODIPY, aiming to expand the utility of such fluorophores along with tailoring their emission properties for high-fidelity bioimaging in vivo. Among these conjugated molecules (Supplementary Figs. 1 and 2), the DCM-IFC fluorophores (covalently integrated fluorescein with chromene) immediately grabbed and focused our attention when we discovered its extremely unusual dualemission. Upon excitation at $480 \mathrm{~nm}$, DCM-IFC (open form, Fig. 2a) displayed two emission peaks at 520 and $700 \mathrm{~nm}$, respectively. When the excitation wavelength was shifted to $560 \mathrm{~nm}$, only the NIR peak at $700 \mathrm{~nm}$ was observed (Fig. 2b). We verified that this unusual dual-emission did not result from contaminations or molecular aggregations by conducting several solvent-dependent and concentration-dependent studies (Supplementary Fig. 3). Clearly, the excitation-dependent emission in DCM-IFC (open form) is not in agreement with Kasha's rule ${ }^{36}$ and warranted further investigation.

We subsequently carried out the experiments with excitation and emission spectra to validate the anti-Kasha mechanism in the open-form DCM-IFC. The fluorescence spectra revealed two emission bands that corresponded to different excitation peaks at $480 \mathrm{~nm}$ and $560 \mathrm{~nm}$, respectively (Fig. 2b). Each excitation peak was mirror-image of their corresponding emission peak, which led us to hypothesize that the two emission bands originated from two different excited states in the open-form DCM-IFC.

Furthermore, quantum chemical calculations were conducted, and their results strongly corroborate the experimental data: the two emission bands matched the fluorescence from the $S_{1}$ (NIR) and $S_{2}$ (visible) states, respectively (Supplementary Table 1 and Supplementary Fig. 4). Electron-hole analysis also indicated that the electron of the $S_{2}$ state has a considerable distribution in the fluorescein moiety, while the electron of the $S_{1}$ state mainly localizes in the DCM fragment (Fig. 2c). Notably, the two excited states are characterized by a large energy gap: $\Delta E\left(\mathrm{~S}_{2}-\mathrm{S}_{1}\right)$ is $0.74 \mathrm{eV}$ (around $6000 \mathrm{~cm}^{-1}$, Supplementary Table 1). This large gap can effectively suppress the internal conversion (IC) from $\mathrm{S}_{2}$ to $S_{1}$ state. Moreover, our calculations indicate that the radiative decay (to $S_{0}$ ) from $S_{2}\left(6.59 \times 10^{8} \mathrm{~S}^{-1}\right)$ is approximately three times faster than that of from $S_{1}\left(1.96 \times 10^{8} \mathrm{~S}^{-1}\right)$. Collectively, these computational results help elucidate the anti-Kasha characteristic which was observed for open-form DCM-IFC.

The anti-Kasha characteristic with dual-emission was also strongly supported by femtosecond time-resolved transient absorption spectroscopic experiments. The time-resolved experiments showed two stimulated emission bands, which is in 
a FRET ratiometric probes (spectral cross-talk, different photobleaching rate)

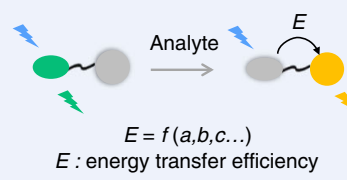

b Unimolecular ratiometric probes (concentration-dependent diffusion)

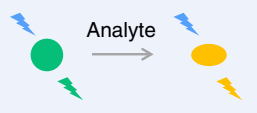

C Anti-Kasha-active ratiometric probes

(accurate quantification assay over space and time)
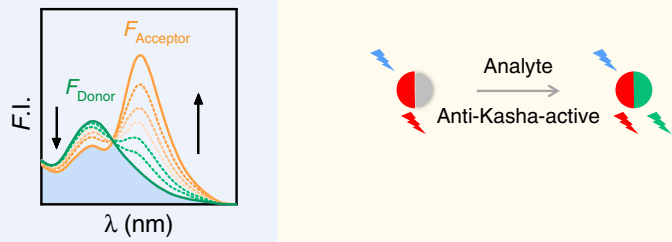

$F_{2}:$ Anti-Kasha-active emission

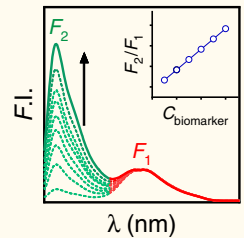

$F_{1}:$ Internal intensity reference

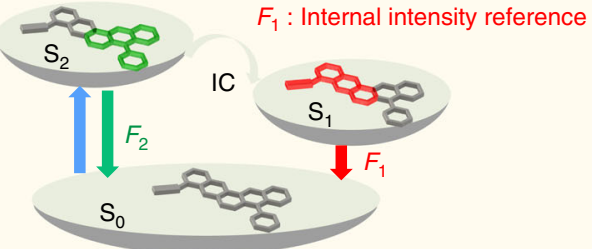

Fig. 1 Strategies for ratiometric probes with characteristic analysis. a FRET ratiometric probes: excitation/emission cross-talk. b Unimolecular ratiometric probes: different photobleaching rates and concentration-dependent diffusion between reactant and product fluorophores in dynamic cellular environment. c In this work, anti-Kasha-active ratiometric probes enabling accurate and reliable ratiometric quantification: an invariant NIR emission from the Kashabased $S_{1}$ state serve as an internal reference; a green emission from the anti-Kasha-active $S_{2}$ state linearly increase with the concentration of targeted analyte, due to the rapid radiative process from $S_{2}$ to $S_{0}$ state, competing with the internal conversion (IC) from $S_{2}$ to $S_{1}$ state.

agreement with our initial hypothesis that the dual-emission stemmed from two discrete excited states (Fig. 2d). The measurement clearly denotes an energy transfer process (IC) from $S_{2}$ to $S_{1}$ state with a lifetime of $\sim 1$ ps (Fig. 2e and Supplementary Table 2); the $\mathrm{S}_{2}$ fluorescence lifetime is also short $(45 \mathrm{ps}$ at $520 \mathrm{~nm})$. According to a general rule ${ }^{26}$, the IC process takes place at least $10^{4}$ times as fast as the spontaneous $S_{n}$ emission. However, DCM-IFC displayed a rapid radiative process from $S_{2}$ to $S_{0}$ state, which implies that the rate of $S_{2}$ emission decay can compete with IC from $S_{2}$ to $S_{1}$ state. These ultra-fast experimental insights led us to propose an intrinsic mechanism of anti-Kasha with dual-emission: a comparable rate between the radiation decay process from $S_{2}$ state and IC to the $S_{1}$ state can account for the observed dual-emission of our anti-Kasha chromophore DCM-IFC (Fig. 2f).

Engineering anti-Kasha/Kasha fluorophores. Although openform DCM-IFC displayed dual-emission with anti-Kasha properties, closed-form DCM-IFC (Fig. 2g) exhibited only a singleemission band at $700 \mathrm{~nm}$ (Fig. 2h). Fluorescence spectra, transient absorption spectra, and quantum chemical calculations (Fig. 2h-k) indicated that closed-form DCM-IFC had emission properties that obeyed Kasha's rule. To further support the discrete properties of open- versus closed-form DCM-IFC, two reference compounds were specifically designed and subsequently synthesized: one compound was structurally locked in an open form, while the other was locked in a closed form. Accordingly, the corresponding reference DCM-IFC-ester clearly exhibited two emission peaks at 520 and $700 \mathrm{~nm}$ in dichloromethane, which strongly confirms the anti-Kasha property to the open form of DCM-IFCs (Fig. 2l, Supplementary Figs. 5-7 and Supplementary Tables 3-4). In contrast, DCM-IFC-ester-R demonstrated the typical single-emission Kasha property (Supplementary Fig. 8). Taken together, these related IFCs strongly confirm the anti-Kasha/Kasha properties in the open/closed forms of DCM-IFCs.

Starting from this initial DCM-IFC molecule, a series of fluorophores were developed based on simple structural modifications using the following guidelines: (i) start with IFC (integrated fluorescein with chromene) backbone as a potential anti-Kasha building block; (ii) introduce an additional electron- withdrawing fluorescence unit (like DCM, TCB, or BI) for extending $\Delta E\left(\mathrm{~S}_{2}-\mathrm{S}_{1}\right)$ (Fig. 3a). To our delight, the open forms of all resulting fluorophores displayed anti-Kasha dual-emission ( $\mathrm{S}_{2}$ emission: $520 \mathrm{~nm}$; $\mathrm{S}_{1}$ emission: 663 to $707 \mathrm{~nm}$; Fig. $3 \mathrm{c}$ and Supplementary Table 5), whereas their closed forms exhibited normal single-emission ( $S_{1}$ emission), complying with Kasha's rule (Fig. 3c).

Subsequent theoretical quantum calculations indicated that the electron distributions for the $S_{2}$ state of these fluorophores (open form) mainly reside in their fluorescein moieties, whereas in the $\mathrm{S}_{1}$ state, the electrons are localized to their electron-withdrawing fluorescence units (e.g., TCB, BI; Supplementary Figs. 9 and 10). Moreover, all these fluorophores have large gap $\Delta E\left(S_{2}-S_{1}\right)$ (Fig. 3c), which is in agreement with a previous report for antiKasha fluorophores with substantial $\mathrm{S}_{2}$ emission ${ }^{37}$. Furthermore, such IFC-integrated fluorophores in their closed forms demonstrated the typical single-emission Kasha band. Fortunately, we acquired a single crystal of reference compound BI-IFC-ester which was absolutely locked in a closed form (Fig. 3b). According to crystal analyses, the conjugated fluorophore combined IFC building block with BI unit exhibits a large coplanar conformation, which is responsible for NIR emission. Compiling these results together, we have successfully developed a generalizable strategy with molecular engineering to extend $\Delta E\left(\mathrm{~S}_{2}-\mathrm{S}_{1}\right)$ gaps that enables construction of diverse anti-Kasha/Kasha chromophores.

Modulating anti-Kasha/Kasha emission for ratiometric mode. To date, it remains very challenging to rationally evoke/regulate emission from higher excited states based on anti-Kasha fluorophores $^{38}$, and these difficulties severely hinder the applicability of such molecules for optical imaging and biosensing. Given that the closed-form DCM-IFC (Kasha fluorophore) and the openform DCM-IFC (anti-Kasha fluorophore) can be reversibly interchanged via the opening and closing of the spirolactone ring (achieved by manipulating $\mathrm{pH}$, Fig. 4a), we hypothesized that rational modulation of the $\mathrm{S}_{2}$ emission could be achieved via antiKasha/Kasha switch.

To verify our hypothesis, we investigated the effect of $\mathrm{pH}$ on the spirolactone ring for the anti-Kasha/Kasha switching of DCM-IFC (Fig. 4b, c). It can be rationalized that a low $\mathrm{pH}$ 
a

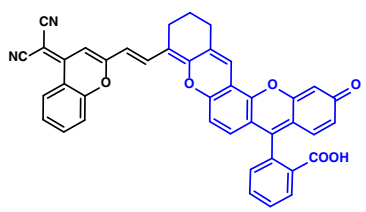

DCM-IFC (open form) Anti-Kasha fluorophore

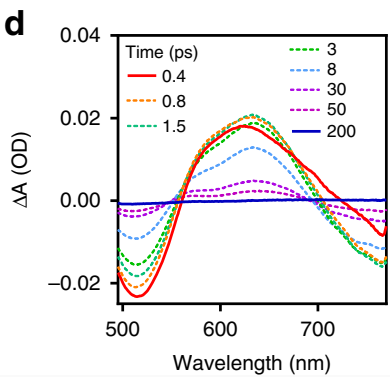

g

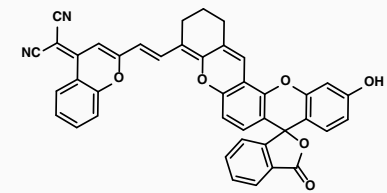

DCM-IFC (closed form) Kasha fluorophore

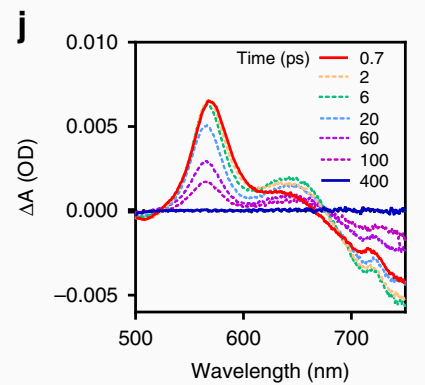

b

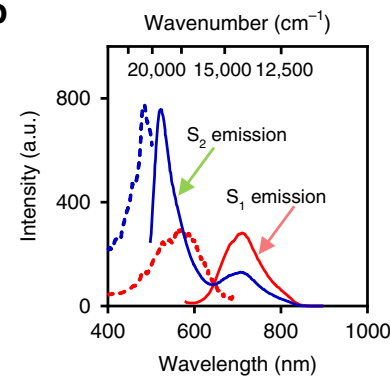

e

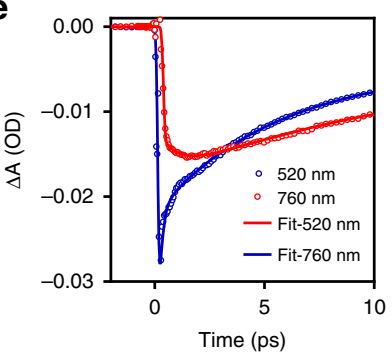

C
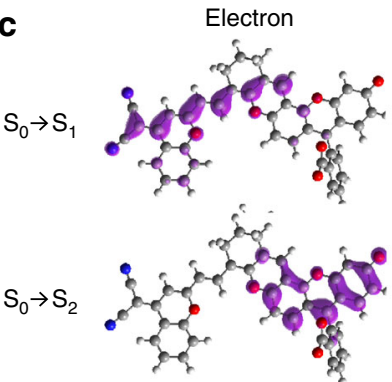

f

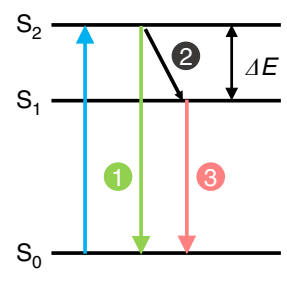

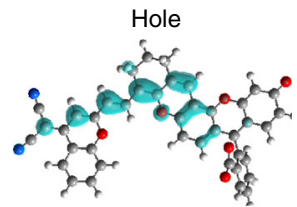

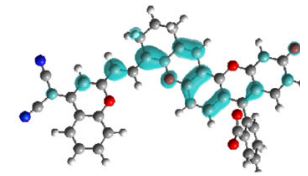$$
\text { . }
$$

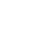

.

h
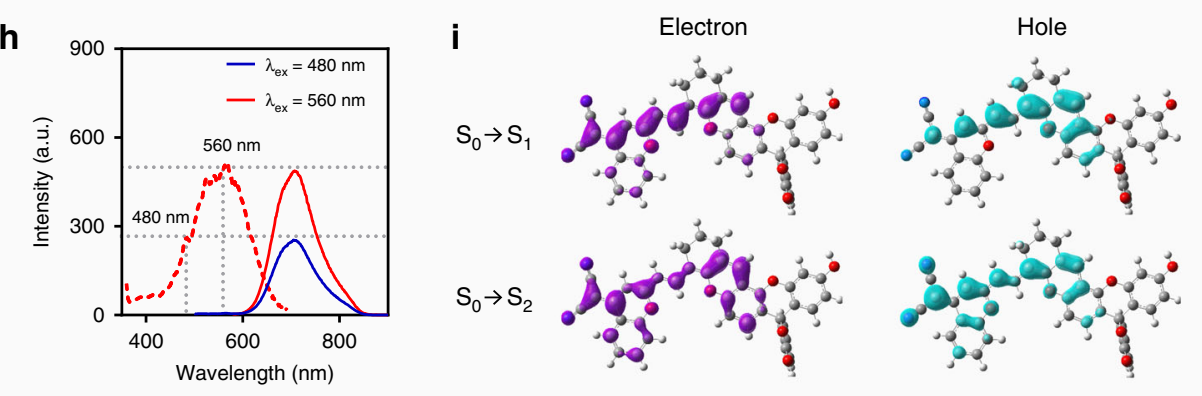

$\mathbf{k}$

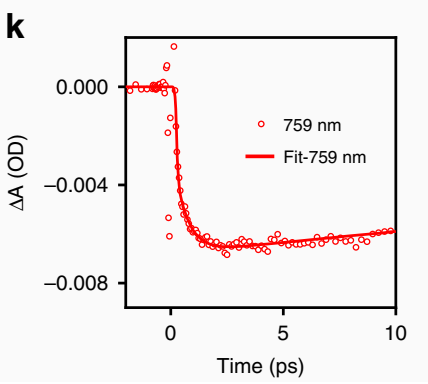

I Reference: locked open form Reference: locked closed form DCM-IFC-ester

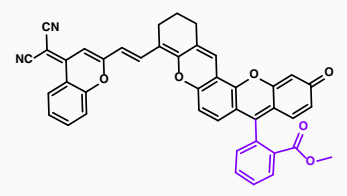

1) Green emission from $S_{2}$ state

2 Internal conversion from $S_{2}$ to $S_{1}$

(3) NIR emission from $S_{1}$ state

Fig. 2 Characterization of anti-Kasha mechanism in open-form versus Kasha mechanism in closed-form. a Chemical structure of DCM-IFC in its open form. b Excitation spectra (dotted line, monitored at $\lambda_{\mathrm{em}}=520$ and $710 \mathrm{~nm}$ ) and emission spectra (solid line, excited at $\lambda_{\mathrm{ex}}=480$ and $560 \mathrm{~nm}$ ) of openform DCM-IFC. c Electron-hole analysis involved during the photoexcitation of the open form, based on the molecular structure at the Franck-Condon state. Femtosecond time-resolved transient absorption spectra (d) and kinetics (e) of open-form DCM-IFC (excited at $\lambda_{\mathrm{ex}}=480 \mathrm{~nm}$ ). $\mathbf{f}$ Jablonski diagram illustrating the anti-Kasha mechanism. $\mathbf{g}$ Chemical structure of DCM-IFC in its closed form. $\mathbf{h}$ Excitation spectra (dotted line, monitored at $\lambda_{\mathrm{em}}=710 \mathrm{~nm}$ ) and emission spectra (solid line, excited at $\lambda_{\mathrm{ex}}=480$ and $560 \mathrm{~nm}$ ) of closed-form DCM-IFC. $\mathbf{i}$ Electron-hole analysis involved during the photoexcitation of the closed form, based on the molecular structure at the Franck-Condon state. Femtosecond time-resolved transient absorption spectra (j) and kinetics (k) of closed-form DCM-IFC (excited at $\lambda_{\mathrm{ex}}=480 \mathrm{~nm}$ ). Note: open-form DCM-IFC was investigated at pH 11.3 and closed-form DCM-IFC was investigated at $\mathrm{pH}$ 2.0. I Two structurally related reference compounds that are locked into open form or closed form. Note: all fluorescence spectra were measured in a mixture solution of acetonitrile $(\mathrm{MeCN}) /$ Britton-Robinson buffer.

environment would induce a spirocyclization reaction that causes the IFC moiety to adopt its closed form. Conversely, increasing $\mathrm{pH}$ values would promote deprotonation and change the IFC moiety into its open form. The solvent $\mathrm{pH}$ was increased from 2.2 to 8.0 and the spirolactone ring-opening was monitored (from Kasha to anti-Kasha): The $S_{2}$ emission at $520 \mathrm{~nm}$ progressively intensifies from an initial zero background. Here the $S_{2}$ emission response clearly established that rational modulation of emission from higher excited states can be achieved by tuning anti-Kasha/ Kasha properties.
To our surprise, the intensity of the $S_{1}$ emission at $700 \mathrm{~nm}$ remains invariable under the tested range of $\mathrm{pH}$ values, which suggests that the Kasha/anti-Kasha dynamics may not influence the $\mathrm{S}_{1}$ emission of DCM-IFC. This response can be explained by quantum chemical calculations and absorbance spectra: (i) calculations showed that the electron density $\left(S_{1}\right.$ state) of the open form and closed form displayed remarkable overlap (Fig. 2c, i), (ii) DCM-IFC displayed similar emission oscillator strength in the $\mathrm{S}_{1}$ state $\left(f_{\text {closed }}=1.9527, f_{\text {open }}=2.1545\right)$ between the closed and the open form since $S_{1}$ state localizes in the DCM moiety and is 

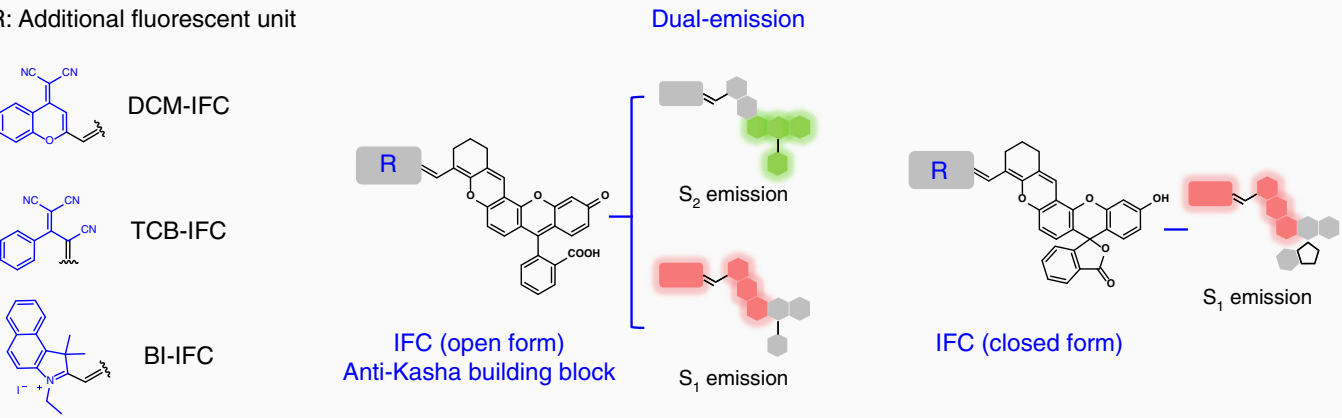
Anti-Kasha building block

IFC (closed form)

b Reference: locked closed form

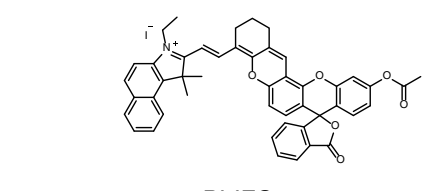

BI-IFC-ester
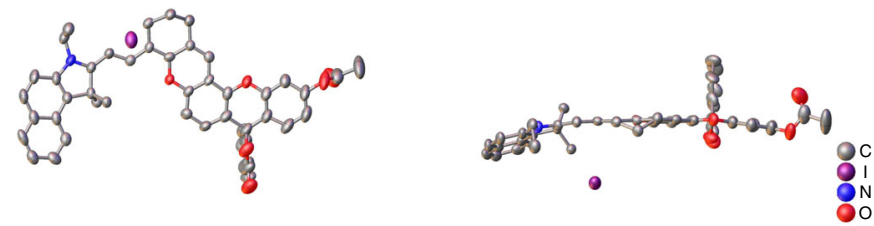

C

\begin{tabular}{|c|c|c|c|c|c|}
\hline \multicolumn{4}{|c|}{ Anti-Kasha } & \multicolumn{2}{|c|}{ Kasha } \\
\hline $\begin{array}{l}\text { Fluorophore } \\
\text { (Open form) }\end{array}$ & $\begin{array}{c}\Delta E\left(\mathrm{~S}_{2}-\mathrm{S}_{1}\right) \\
(\mathrm{eV})\end{array}$ & $\begin{array}{l}\text { Oscillator } \\
\text { Strength }\end{array}$ & $\begin{array}{c}\text { Emission } \\
\qquad(\mathrm{nm})\end{array}$ & $\begin{array}{l}\text { Fluorophore } \\
\text { (Closedform) }\end{array}$ & $\begin{array}{c}\text { Emission } \\
(\mathrm{nm})\end{array}$ \\
\hline DCM-IFC & 0.74 & $\begin{array}{ll}S_{2} & 0.2557 \\
S_{1} & 2.1545\end{array}$ & $\begin{array}{l}523 \\
707\end{array}$ & DCM-IFC & 707 \\
\hline TCB-IFC & 0.64 & $\begin{array}{ll}S_{2} & 0.3146 \\
S_{1} & 1.6792\end{array}$ & $\begin{array}{l}524 \\
663\end{array}$ & TCB-IFC & 663 \\
\hline $\mathrm{BI}-\mathrm{IFC}$ & 0.74 & $\begin{array}{ll}S_{2} & 0.3625 \\
S_{1} & 1.7558\end{array}$ & $\begin{array}{l}522 \\
685\end{array}$ & BI-IFC & 685 \\
\hline
\end{tabular}

Fig. 3 A generalizable molecule engineering strategy for developing anti-Kasha/Kasha fluorophores. a Schematic illustration of designing anti-Kasha/ Kasha fluorophores. b Molecular and crystal structures of BI-IFC-ester (locked closed form). c Quantum chemical calculations and experimental data of anti-Kasha/Kasha fluorophores. $\Delta E\left(\mathrm{~S}_{2}-\mathrm{S}_{1}\right)$ and oscillator strength were obtained from quantum chemical calculations. Emission was obtained from experimental data.

relatively unaffected by the spirocyclization of the fluorescein moiety, and (iii) the enhanced emission at $520 \mathrm{~nm}$ may originate from DCM-IFC's increased photoreceptivity of the open vs. closed form as the $\mathrm{pH}$ changes (Fig. $4 \mathrm{~b}, \mathrm{c}$ ).

The combination of variable $S_{2}$ anti-Kasha emission with nearly invariant $S_{1}$ Kasha emission alludes the possibility that our IFCs could be exploited as unimolecular probes for ratiometric imaging and for quantification applications. We envisioned that $\mathrm{S}_{1}$ emission could act as an internal intensity reference, while the $\mathrm{S}_{2}$ emission would reliably respond and reflect $\mathrm{pH}$ changes. As expected, the spirolactone-switch-induced dual-emission response with a constant internal intensity reference was exhibited by all of the IFC-derived chromophores (Fig. $4 \mathrm{~d}-\mathrm{f}$ and Supplementary Figs. 11 and 12). In addition, the ratio $\left(\mathrm{S}_{2} / \mathrm{S}_{1}\right)$ of dual-emission remained stable across varying probe concentrations $(5-30 \mu \mathrm{M})$, indicating that the observable ratio $\left(S_{2} / S_{1}\right)$ of IFC-derived chromophores is completely concentrationindependent (Fig. 4g, h). Subsequently, as a proof-of-concept, we investigated the applicability of dual-emission IFC probes for monitoring $\mathrm{pH}$ changes in living cells (Fig. 4i-k). Overall, we have successfully developed and demonstrated a viable strategy for ratiometric analysis with an internal intensity reference signal based on modulation of the anti-Kasha/Kasha properties.
Ratiometric quantitative sensing over space and time. Given that IFC chromophores mainly remain in their open form, and emit both $S_{2}$ and $S_{1}$ fluorescence signals at the physiological $\mathrm{pH}$ range of 7.0-8.0 (Fig. 4c-f), we hypothesized that replacing the hydroxy group in IFCs with different biorecognition units can absolutely lock such probes in their closed form and emit only NIR fluorescence $\left(S_{1}\right)$. Upon encountering their corresponding analyte, the hydroxyl group would be restored, thereby turning the probe to its open form at the desirable physiological $\mathrm{pH}$, which would emit both green $\left(S_{2}\right)$ and NIR $\left(S_{1}\right)$ fluorescence (Fig. 5a). By calculating the ratio of $S_{2}$ and $S_{1}$ emission, it is thus possible to determine the concentration of the target analyte. In this regard, various ratiometric fluorescent probes could be built up via using our specific IFC chromophores.

Consequently, the following functionalized IFC probes were synthesized: DCM-IFC-1 with an acrylate group for sensing cysteine (Cys), DCM-IFC-2 with 2,4-dinitrobenzenesulfonyl for sensing glutathione $(\mathrm{GSH})^{39,40}$, as well as DCM-IFC-3 and DCMIFC- 4 with a disulfide bond that can sense GSH and then release the anticancer drug camptothecin $(\mathrm{CPT})^{41,42}$ (Fig. 5b, f, j). As shown in Fig. $5 \mathrm{c}, \mathrm{g}, \mathrm{k}$, a ratiometric response with the internal intensity reference signal (linearly increasing $S_{2}$ emission intensity, stable $S_{1}$ emission) was observed in all test assays at 
a
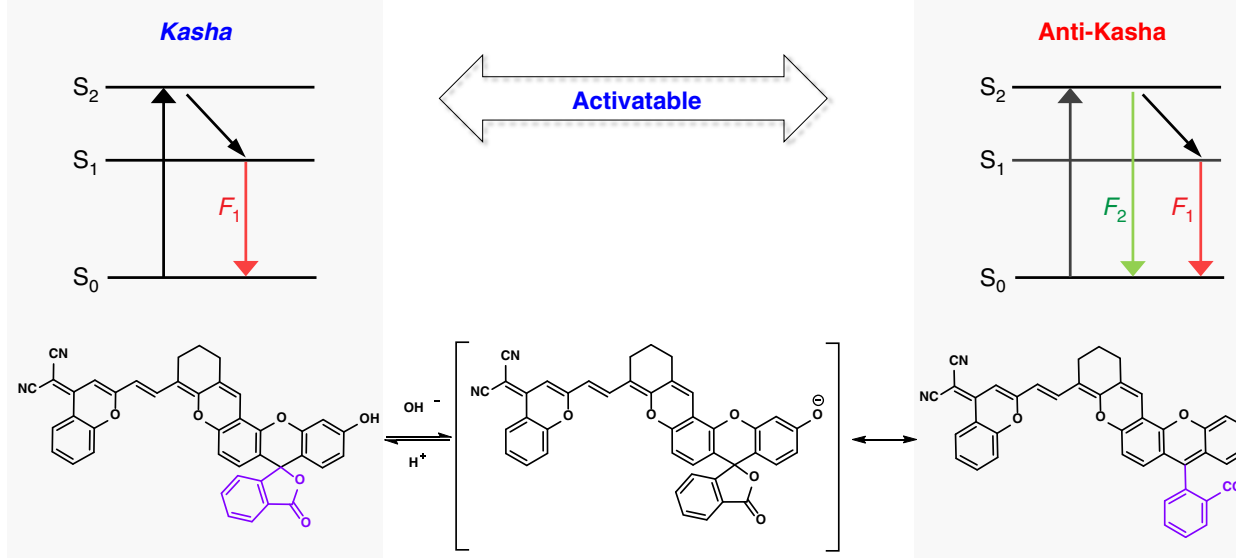

Ring-closed form
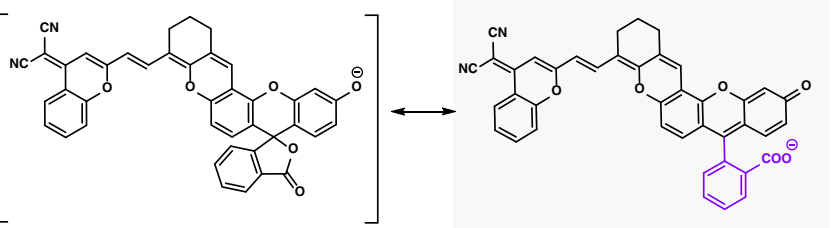

Ring-open form b
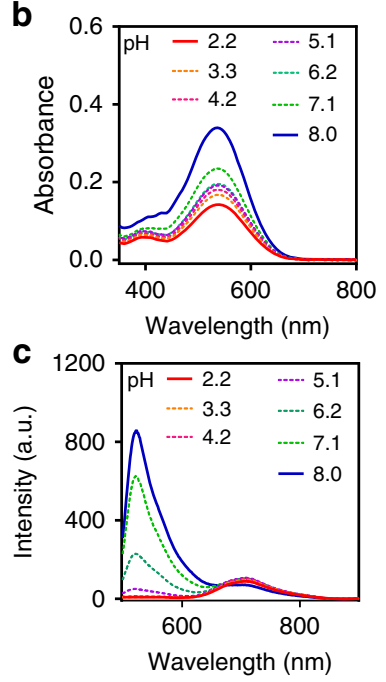
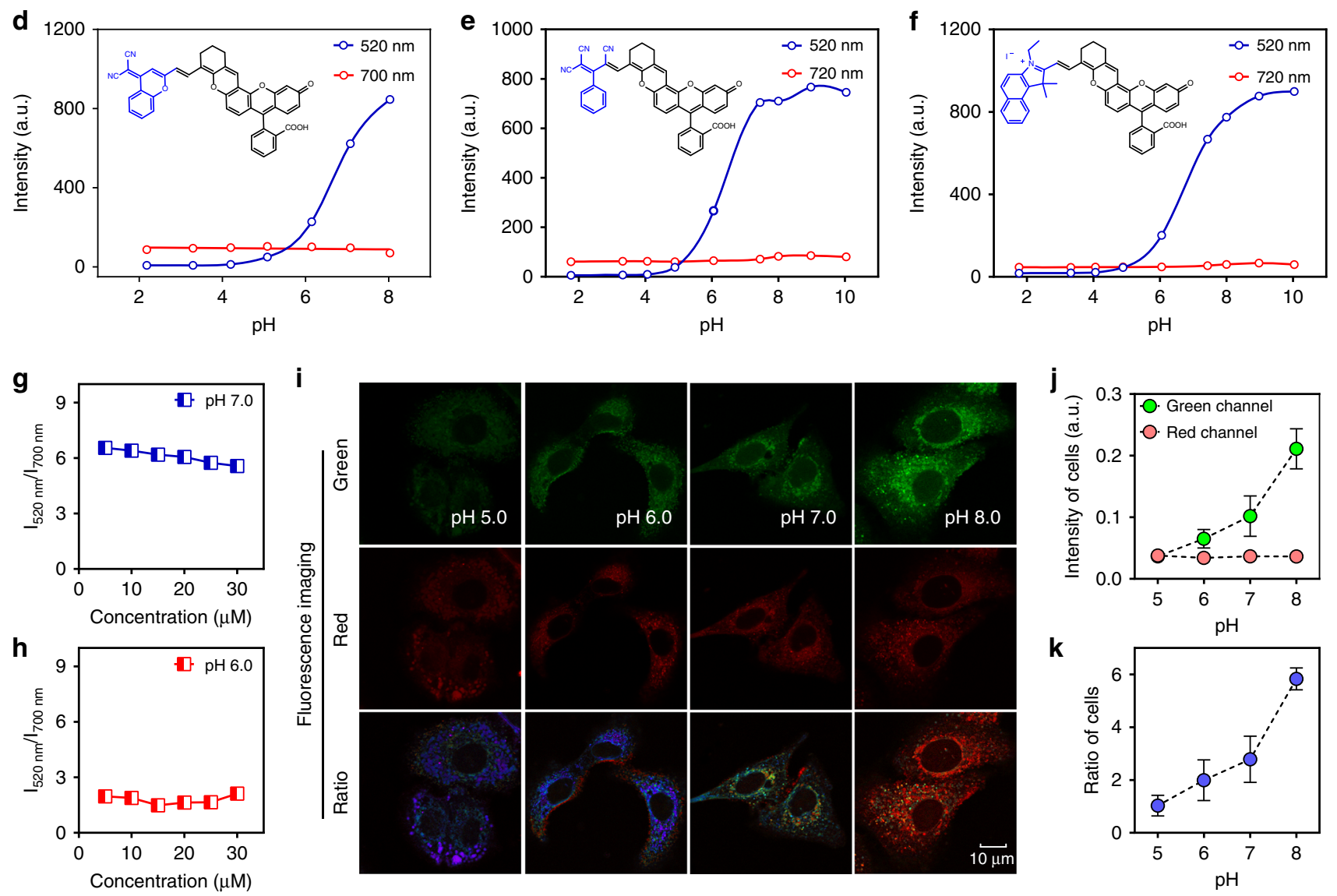

$\mathbf{k}$

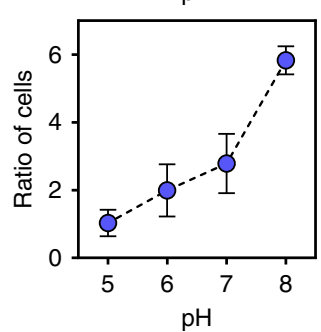

Fig. 4 Anti-Kasha/Kasha switch-induced dual-emission response with an internal intensity reference signal. a Schematic illustration of spirolactoneswitch-controlled molecules for tuning anti-Kasha/Kasha properties. Absorption (b) and fluorescence spectra (c) of DCM-IFC in the range of pH 2.2-8.0. Dual-emission response with the internal intensity reference signal of DCM-IFC (d), TCB-IFC (e), and BI-IFC (f) at different pH values, $\lambda$ ex $=480 \mathrm{~nm}$. Source data are provided as a Source Data file. Concentration-dependent emission ratios $\left(\mathrm{S}_{2} / \mathrm{S}_{1}\right)$ detected for $\mathrm{DMC}-\mathrm{IFC}$ at $\mathrm{pH} 7.0(\mathbf{g})$ and $\mathrm{pH} 6.0(\mathbf{h})$. Source data are provided as a Source Data file. Dual-channel ratiometric imaging (i), fluorescence intensity (j) and ratio (k) of DCM-IFC in A549 cells across different $\mathrm{pH}$ values. Data with error bars are expressed as mean \pm s.d., $n=3$. Source data are provided as a Source Data file. Note: all fluorescence spectra were measured in a mixture solution of $\mathrm{MeCN} /$ Britton-Robinson buffer.

desirable physiological pH condition. For example, with probe DCM-IFC-1, the fluorescence signal at $520 \mathrm{~nm}$ increased linearly with the concentration of Cys, while the emission at $700 \mathrm{~nm}$ remained nearly constant (Fig. 5d). The intensity ratios at these two wavelengths correlated linearly with the concentrations of
Cys (Fig. 5e). Similar emission profiles and ratiometric correlations were also observed in DCM-IFC-2, DCM-IFC-3 and DCMIFC-4 towards GSH (Fig. 5h, i, l, m). These results (Fig. 5 and Supplementary Figs. 13-26) showed that the IFC platform provided a generalizable method for quantitative analysis of 


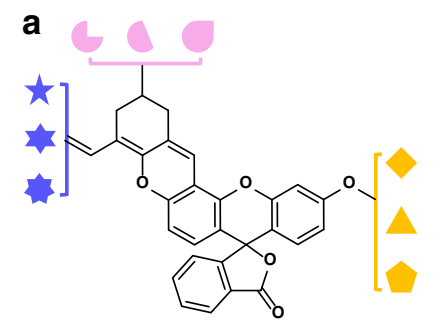

Ring-closed form IFC Probes

b

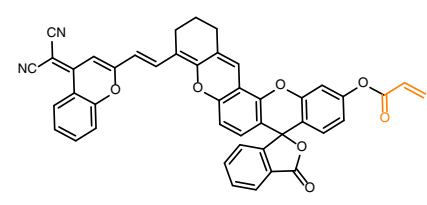

DCM-IFC-1

$\mathbf{f}$

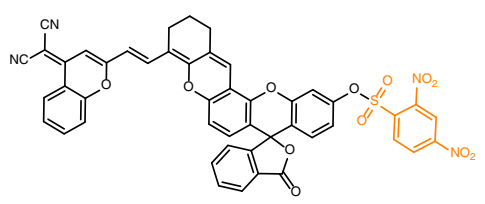

DCM-IFC-2

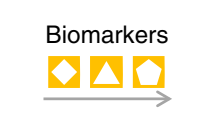

At physiological $\mathrm{pH}$

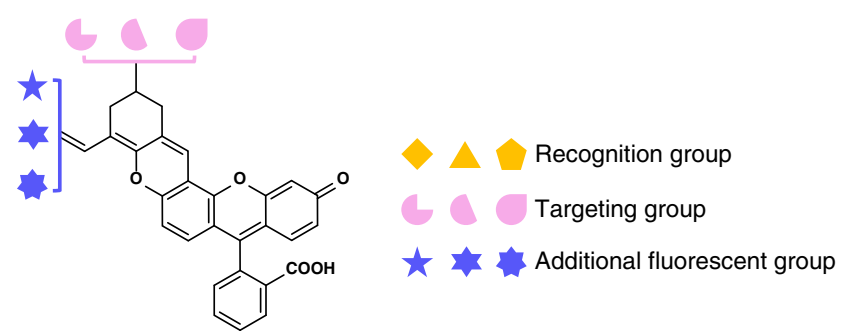

Ring-open form

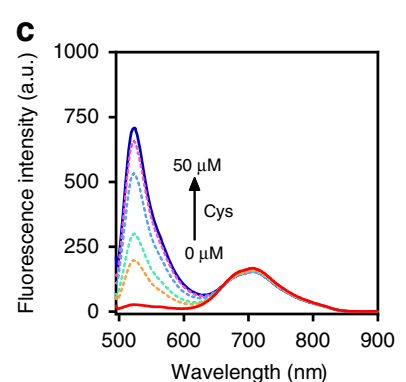

g
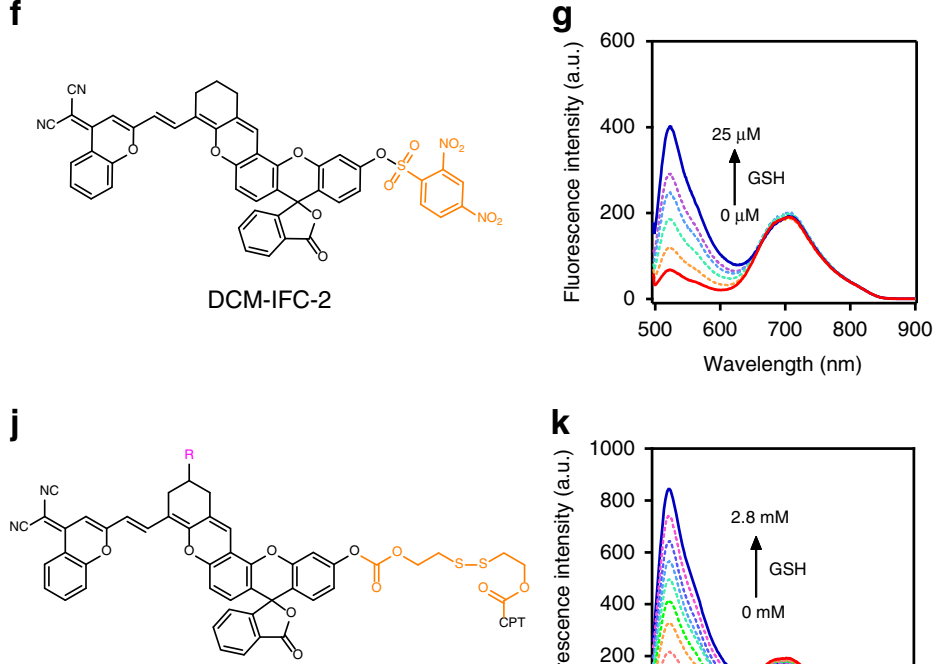

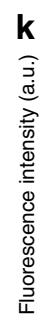

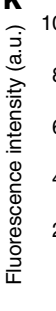

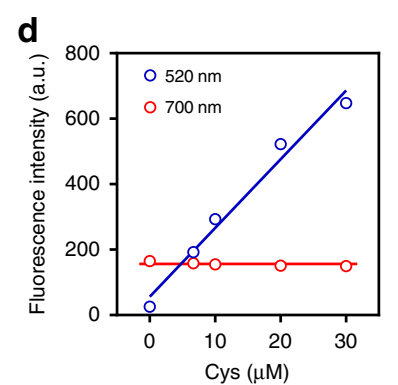

h

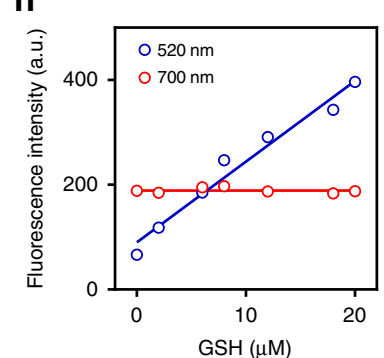

I

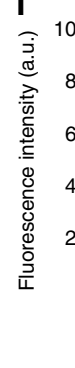

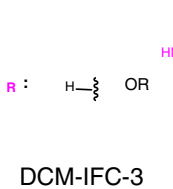

DCM-IFC-3

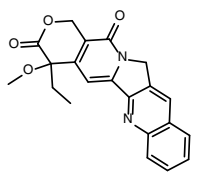

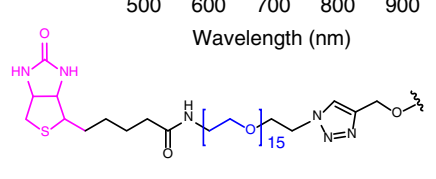

DCM-IFC-4

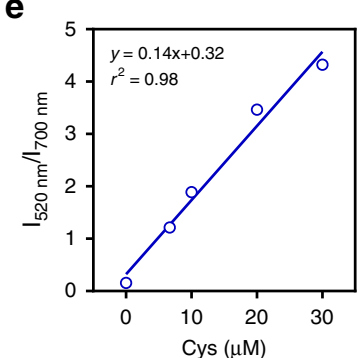

i

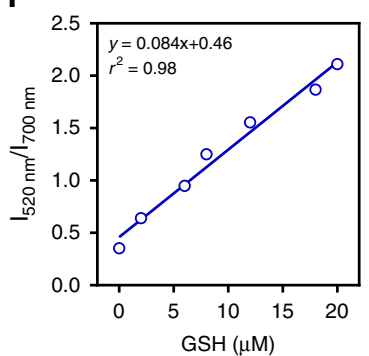

m

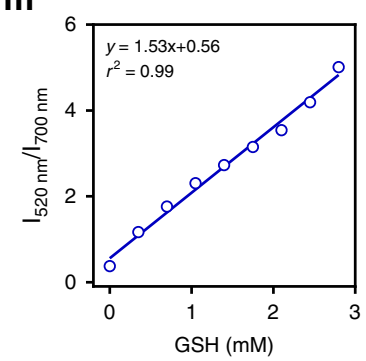

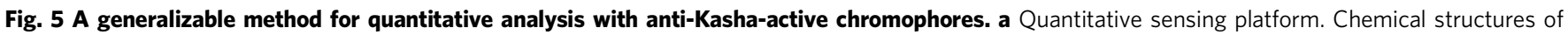

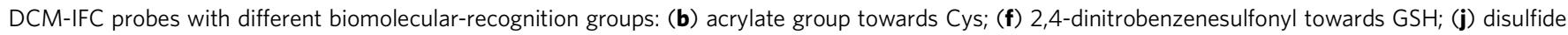

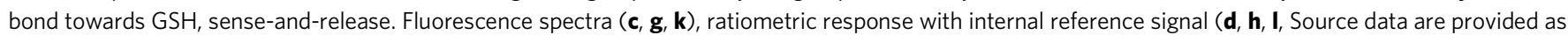

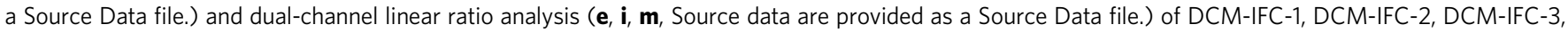
and DCM-IFC-4 for various analytes. Note: all fluorescence spectra were measured in a mixture solution of MeCN/phosphate-buffered saline (PBS,

$\mathrm{pH}=7.4), \lambda_{\mathrm{ex}}=480 \mathrm{~nm}$.

various biomolecules via ratiometric imaging using activatable anti-Kasha $S_{2}$ emission and invariant $S_{1}$ emission as a stable reference signal.

The next step involved the use of our anti-Kasha-active chromophores for quantitative analysis of endogenous biomolecules in living systems. To establish the correlation between the GSH concentration and the ratio $\left(\mathrm{S}_{2} / \mathrm{S}_{1}\right)$, we pretreated QSG-7701 cells with excessive $\mathrm{N}$-methylmaleimide (NEM, a derivative known to covalently sequester $\mathrm{GSH}, 500 \mu \mathrm{M}$ ) for $30 \mathrm{~min}$ to remove cellular GSH (Fig. 6a-d). After removing the NEM, the pretreated cells were then incubated with different doses of GSH for another $30 \mathrm{~min}$, followed by further incubation in $30 \mu \mathrm{M}$ of DCM-IFC-4 for $60 \mathrm{~min}$. It is noteworthy that the intensity of the green channel $\left(\mathrm{S}_{2}\right.$ emission, $\left.520 \pm 20 \mathrm{~nm}\right)$ gradually increased with the GSH concentration, while that of the red channel ( $\mathrm{S}_{1}$ emission, $700 \pm 20 \mathrm{~nm}$ ) remained nearly constant (Fig. 6i), which is consistent with previous experiments in vitro (Fig. 5). Moreover, the ratio of resulting fluorescence intensity between 

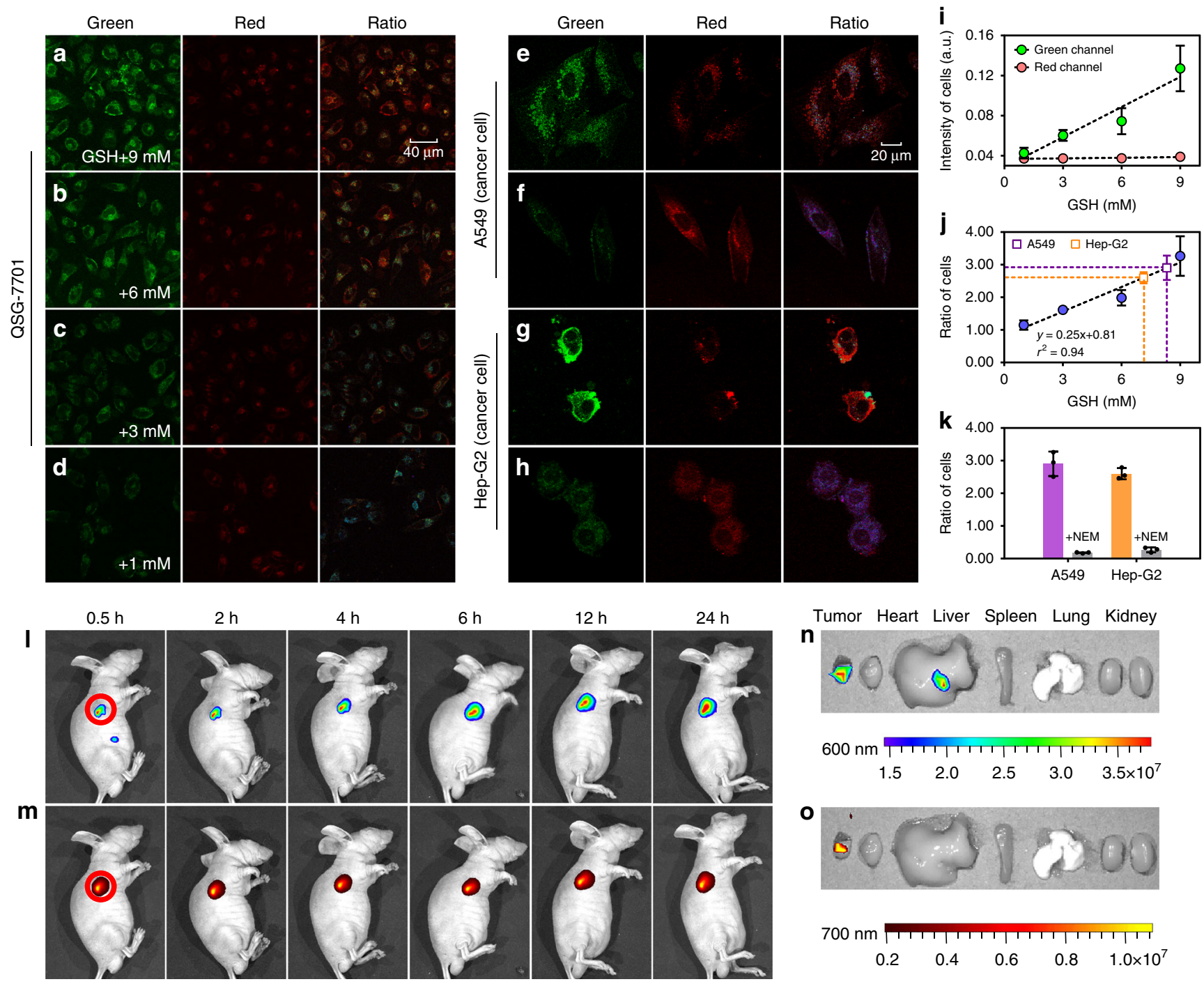

Fig. 6 Quantitative sensing in live cells and dual-emission imaging in vivo. Dual-channel and ratiometric imaging of QSG-7701 cells (a-d, pretreated with various concentrations of GSH); A549 and Hep-G2 cells incubated with DCM-IFC-4 (30 $\mu \mathrm{M})$ and either untreated (e, $\mathbf{g})$ or treated with (f, h) NEM (a derivatization agent which covalently sequesters GSH). Note: The green channel was $520 \pm 20 \mathrm{~nm}$, the red channel was $700 \pm 20 \mathrm{~nm}$, and ratiometric images were generated from the 520 and $700 \mathrm{~nm}$ channels, $\lambda_{\mathrm{ex}}=488 \mathrm{~nm}$. i Dual-channel response with internal reference signal in cells. Data with error bars are expressed as mean \pm s.d., $n=3$. Source data are provided as a Source Data file. $\mathbf{j}$ Standard curve of the $I_{520 \mathrm{~nm}} I_{700 \mathrm{~nm}}$ ratio as a function of GSH concentration. Data with error bars are expressed as mean \pm s.d., $n=3$. Source data are provided as a Source Data file. $\mathbf{k}$ Ratio value in A549 and Hep-G2 cells with and without NEM. Data with error bars are expressed as mean \pm s.d., $n=3$. Source data are provided as a Source Data file. (I, $\mathbf{m}$ ) In vivo dualchannel fluorescence imaging of xenograft tumor (A549 cell) bearing mice at various times $(0.5,2,4,6,12,24 \mathrm{~h}$ ) after the intravenous injection of DCMIFC-4 (2.44 $\left.\mathrm{mg} \mathrm{kg}^{-1}\right)$, the tumor site is circled in red. $\mathbf{n}, \mathbf{o}$ Ex vivo dual-channel fluorescence imaging of the excised organs (tumor, heart, liver, spleen, lung, and kidney) at $24 \mathrm{~h}$ after the intravenous injection of DCM-IFC-4. Note: fluorescence signals at $600 \mathrm{~nm}$ (rainbow scale) and $700 \mathrm{~nm}$ (yellow-red scale).

the green channel and the red channel demonstrated a strong linear correlation with the GSH concentration $\left(r^{2}=0.94\right.$, Fig. $\left.6 j\right)$. This correlation can be used to generate a calibration curve that enables the precise determination of GSH concentration in cells simply by using reliable ratiometric imaging.

Subsequently, we used DCM-IFC-4 to quantify GSH concentration in GSH-enriched cells from two tumor cell lines ${ }^{43}$ (human lung cancer cells A549 and human hepatic cancer cells Hep-G2, Fig. 6e-h). Under identical experimental conditions, A549 cells and Hep-G2 cells exhibited intensity ratios of 2.90 and 2.59, respectively (Fig. 6j). By extrapolating the calibration curve, we calculated the GSH concentrations in A549 cells $(8.36 \mathrm{mM})$ and in Hep-G2 cells $(7.12 \mathrm{mM})$. These concentrations are in good agreement with previously reported GSH concentration values ${ }^{44}$. Furthermore, upon exposure of both cell lines with NEM, we observed a sharp decrease in the green channel emission and the associated intensity ratio between the green and red channels (Fig. 6f, h, k). These experiments helped to establish how our anti-Kasha-active fluorophores enable quantitative ratiometric analysis of endogenous biomolecules in living cells.

In addition to quantitative analysis of biomolecules in cells, our anti-Kasha IFC platform also realized a "sense-and-release" activity for targeted drug delivery. An ideal targeted drug delivery system should allow accurate pathologic diagnosis of a given cell's state and then precisely deliver said drug at its optimal dosage, thus achieving personalized theragnostics. However, owing to a dearth of reliable quantitative analytical methods, it remains largely difficult for the controlled release of dosages. To address this issue, we designed and synthesized DCM-IFC-4 that contains two functional groups. One of the functional groups 
is a hydrophilic PEG oligomer-bridged biotin segment, responsible for active targeting of tumor cells ${ }^{45}$. The other is a hydrophobic disulfide-bridged anticancer prodrug CPT (Supplementary Fig. 27). Upon DCM-IFC-4's interaction with GSH, the CPT drug is released. High-performance liquid chromatography (HPLC) experiments showed that the CPT release dosages were linearly dependent on GSH concentrations (Supplementary Fig. 28). These results serve to demonstrate a controlled way of releasing drug dosages based on our designed DCM-IFC motif.

Finally, we explored the use of DCM-IFC-4 for selectively sensing biomolecules and releasing CPT in A549 tumor-bearing mice. After the intravenous injection of DCM-IFC-4 $\left(2.44 \mathrm{mg} \mathrm{kg}^{-1}\right)$ to A549 xenograft tumor-bearing mice, in vivo and ex vivo fluorescence bioimaging was performed via a dual read-out channel: $600 \pm 20 \mathrm{~nm}$ (Fig. 6l) and $700 \pm 20 \mathrm{~nm}$ (Fig. 6m). The $700 \mathrm{~nm}$ emission signal was indicative of specific probe accumulation in tumors, and here the observed specificity can be attributed to the synergistic passive (a preferable micelle-based EPR effect) and active (biotin receptor-mediated endocytosis) targeting abilities. The emission intensity at $600 \mathrm{~nm}$ gradually increased throughout the treatment window period. To verify that the fluorescence signals were only caused by probe accumulation in the tumor cells, we recorded fluorescence images of ex vivo tumor tissue and major organs (Fig. 6n, o). Ex vivo data showed that only the tumor tissue displayed a strong fluorescence signal, exactly in good agreement with the in vivo results. These imaging results further highlight the potential of DCM-IFC-4 for in vivo quantification of biomolecules in targeted cell types, as well as for synergistic tumor targeting and controlled drug release strategies.

\section{Discussion}

There are well-known limitations with fluorescence-based technologies for cellular imaging, and especially for the inevitable excitation/emission cross-talk in FRET mode. Another important limitation of the current analytical strategy is the lack of a constant signal for monitoring and measuring biomolecules which can be used as an internal intensity reference. Indeed, this lack is widely recognized among the FRET community, and all manner of workarounds have been proposed and employed to overcome this fundamental deficiency. Some groups have adopted unimolecular probes in their efforts to overcome challenges to accurate quantification in dynamic cellular environment, but still suffer from inherent limitations particularly, including errors from environmental sensitivity and concentration-dependent diffusion properties of dual emission between reactant and product fluorophores.

The development of anti-Kasha chromophores with dualemission properties and a stable internal intensity reference signal simultaneously addresses major challenges to limited quantitative analysis of biomolecules in dynamic cellular environment. Fundamentally, the essential enabling innovation of our study is aimed at evoking/regulating emission from higher excited states based on anti-Kasha fluorophores, whereas almost all molecules exhibit only single emission according to the so-called Kasha's rule. In particular, given the exceedingly rare anti-Kasha dyes, it is a long-term goal for scientists that unimolecular fluorophores with discrete excitation states and attendant Kasha vs. anti-Kasha emission, exhibit dual-emission spectra behaviors. Notably, this elaboration of the underlying design principle to generate ratiometric probes can construct and exert the reliable quantitative calibration for various biomolecules, not affected by extraneous factors including probe concentrations, excitation power, and cellular context.
In summary, we focused on engineering long-wavelength antiKasha/Kasha fluorophores for reliable ratiometric quantification of biomolecules in a physiological context. We have successfully developed a generalizable strategy with molecular engineering to extend $\Delta E\left(\mathrm{~S}_{2}-\mathrm{S}_{1}\right)$ gaps that enables construction of diverse antiKasha/Kasha chromophores via introducing an additional electron-withdrawing fluorescence unit to integrated fluorescein with chromene (IFC) building block. With the quantum chemical calculations and femtosecond time-resolved transient absorption spectroscopic experiments, the IFC-based fluorophores display a rapid radiative process from $S_{2}$ to $S_{0}$ state, and can compete with the IC from $S_{2}$ to $S_{1}$ state, thereby accounting for the intrinsic anti-Kasha mechanism with dual-emission: an invariant NIR emission from the Kasha-based $S_{1}$ state and a green emission at around $520 \mathrm{~nm}$ from the anti-Kasha-based $\mathrm{S}_{2}$ state. The combination of the dual-emission and invariant NIR internal reference in these fluorophores allows construction of various probes that can be applied for reliable ratiometric bioimaging and quantitative sensing of biomolecules (such as GSH and Cys) at the desirable physiological $\mathrm{pH}$ range of 7.0-8.0, as well as monitoring targeted drug release, both in vitro and in vivo.

Our work represents a ratiometric sensing platform based on anti-Kasha activatable chromophores, and its applicability in accurately quantifying multiple parameters with concentrationindependence over space and time in biological and medical research. We anticipate that our strategy of tuning anti-Kasha/ Kasha characteristics would inspire the creation of a generation of quantitative metabolic assays in a physiological context, thus greatly expanding the bio-analytical toolboxes for both basic life science research and clinical applications.

\begin{abstract}
Methods
Materials and general methods. Unless special stated, all solvents and chemicals were purchased from commercial suppliers in analytical grade and used without further purification. The ${ }^{1} \mathrm{H}$ and ${ }^{13} \mathrm{C}$ NMR spectra were recorded on a Bruker $\mathrm{AM}$ 400 spectrometer, using TMS as an internal standard. High resolution mass spectrometry data were obtained with a Waters LCT Premier XE spectrometer. UV-vis absorption spectra were collected on a Varian Cary 500 spectrophotometer, and fluorescence spectrum measurements were performed on a Varian Cary Eclipse fluorescence spectrophotometer. Femtosecond time-resolved transient absorption spectra were recorded using HELIOS Fire Femtosecond Transient Absorption Spectrometer. Particle size was measured by dynamic light scattering (DLS) with a NICOMP 380 ZLS. Transmission electron microscopy (TEM) images were obtained on a JEOL 100CX transmission electron microscope operating at an accelerating bias voltage of $100 \mathrm{kV}$. HPLC analysis was performed on an Agilent 1100 series. Confocal fluorescence images were taken on a Leica TCS SP8 $(63 \times$ oil lens). In vivo fluorescence images were measured with a PerkinElmer IVIS Lumina Kinetic Series III imaging system. Synthesis methods for all compounds, characterization data are provided in the Supplementary Information.
\end{abstract}

Theoretical calculation details. Density functional theory (DFT) and timedependent (TD)-DFT calculations were performed using Gaussian $16^{46}$. Unless stated otherwise, geometry optimizations of all molecules in the ground $\left(\mathrm{S}_{0}\right)$ and excited state $\left(S_{1}\right.$ and $\left.S_{2}\right)$ were performed using the M062X functional and the def2SVP basis set in water ${ }^{47}$. Solvent effects were taken in account using the SMD model ${ }^{48}$. Frequency checks were carried out after each geometry optimization to ensure that the minima on the potential energy surfaces (PESs) were found. The radiation rates and non-radiation rates of compounds in aqueous solution were predicted using the MOlecular MAterials Property Prediction Package (MOMAP) 1.0 developed by Shuai group ${ }^{49}$. During the quantum yield prediction for open form, we had replaced the carboxyl phenyl ring with a hydrogen atom for two reasons: (1) MOMAP tends to overestimate the non-radiation rate associated with the slight rotation of the carboxyl phenyl ring upon photoexcitation, while this ring had little contribution to the UV-vis absorption and fluorescence properties of molecules (open form). (2) This replacement reduces computational load.

Crystallography. CCDC 1909724 (BI-IFC-ester) contain the supplementary crystallographic data for this paper. These data can be obtained free of charge from the Cambridge Crystallographic Data Centre via www.ccdc.cam.ac.uk/ data_request/cif. 
Cell lines. The cell lines were purchased from the Institute of Cell Biology (Shanghai, China). Cells were all propagated in T-75 flasks cultured at $37^{\circ} \mathrm{C}$ under a humidified $5 \% \mathrm{CO}_{2}$ atmosphere in RPMI-1640 medium or DMEM medium (GIBCO/Invitrogen, Camarillo, CA, USA), which were supplemented with $10 \%$ fetal bovine serum (FBS, Biological Industry, Kibbutz Beit Haemek, Israel) and 1\% penicillin-streptomycin $\left(10,000 \mathrm{U} \mathrm{mL}^{-1}\right.$ penicillin and $10 \mathrm{mg} \mathrm{mL}^{-1}$ streptomycin, Solarbio life science, Beijing, China).

In vitro cellular imaging. The cells at $1 \times 10^{5}$ cells/well were seeded onto glassbottom petri dishes with complete medium $(1.5 \mathrm{~mL})$ for $12 \mathrm{~h}$. Then the cells preincubated with and without GSH or NEM were exposed to desired concentrations of compounds for $0.5 \mathrm{~h}$. PBS was used to wash cells for three times to clean the background. Four percent paraformaldehyde was added at room temperature for $20 \mathrm{~min}$. The fixed cells were rinsed with PBS twice. The images were then photographed by using a confocal laser scanning microscope Leica TCS SP8 (63x oil lens)

Animals. All animal studies were approved by the Animal Care and Use Committee in accordance with the guidelines for the care and use of laboratory animals. The 3-4-week-old female BALB/cA nude mice were purchased from Shanghai Genechem Co.,Ltd., and maintained under standard conditions. The animals were housed in sterile cages within laminar airflow hoods in a specific pathogen-free room with a 12-h light/12-h dark schedule and fed autoclaved chow and water ad libitum. Production Permit No.: SCXK (Shanghai) 2013-0017. SYXK No. of Shanghai Institute of Materia Medica: SYXK (Shanghai) 2013-0049.

Real-time in vivo imaging in tumor-bearing mice. The nude mice were inoculated with A549 cells on their right flanks by injecting $10^{6}$ cells subcutaneously. When the tumors grew up to $10 \mathrm{~mm}$ in diameter, DCM-IFC-4 in PBS were intravenously injected via tail vein into the A549 cell tumor-bearing nude mice. The real-time in vivo imaging was recorded at different time internals after DCMIFC-4 injection. In vivo fluorescence images were measured with a PerkinElmer IVIS Lumina Kinetic Series III imaging system. After injection, the mice were sacrificed at $24 \mathrm{~h}$. The grafted tumor tissues and major organs, including kidney, lung, spleen, liver, heart were excised and washed with $0.9 \%$ saline. The optical images of the organs and tissues were taken using a PE in vivo Professional Imaging System as described above.

Reporting summary. Further information on research design is available in the Nature Research Reporting Summary linked to this article.

\section{Data availability}

The X-ray crystallographic coordinates for structures reported in this study have been deposited at the Cambridge Crystallographic Data Centre (CCDC), under deposition numbers 1909724. These data can be obtained free of charge from The Cambridge Crystallographic Data Centre via www.ccdc.cam.ac.uk/data_request/cif. The source data underlying Fig. 4d-h, j, k, 5d, e, h, i, l, m, and 6i-k, as well as Supplementary Figs. 15, 17, $24,26,31,32,34 \mathrm{f}$ and Supplementary Table 7 are provided as a Source Data file. All other data are available from the corresponding author.

Received: 21 September 2019; Accepted: 9 January 2020; Published online: 07 February 2020

\section{References}

1. Sun, W., Guo, S., Hu, C., Fan, J. \& Peng, X. Recent development of chemosensors based on cyanine platforms. Chem. Rev. 116, 7768-7817 (2016).

2. Gao, G. et al. Spiro-fused perylene diimide arrays. J. Am. Chem. Soc. 139 , 15914-15920 (2017).

3. Sprick, R. S. et al. Tunable organic photocatalysts for visible-light-driven hydrogen evolution. J. Am. Chem. Soc. 137, 3265-3270 (2015).

4. Zhao, T. et al. A transistor-like $\mathrm{pH}$ nanoprobe for tumour detection and image-guided surgery. Nat. Biomed. Eng. 1, 0006 (2016).

5. Chan, J., Dodani, S. C. \& Chang, C. J. Reaction-based small-molecule fluorescent probes for chemoselective bioimaging. Nat. Chem. 4, 973-984 (2012).

6. Hong, G., Antaris, A. L. \& Dai, H. Near-infrared fluorophores for biomedical imaging. Nat. Biomed. Eng. 1, 0010 (2017).

7. Qi, J. et al. Light-driven transformable optical agent with adaptive functions for boosting cancer surgery outcomes. Nat. Commun. 9, 1848 (2018).

8. Green, O. et al. Near-infrared dioxetane luminophores with direct chemiluminescence emission mode. J. Am. Chem. Soc. 139, 13243-13248 (2017).
9. Grimm, J. B. et al. A general method to fine-tune fluorophores for live-cell and in vivo imaging. Nat. Methods 14, 987-994 (2017).

10. Zheng, X. C. et al. Successively activatable ultrasensitive probe for imaging tumour acidity and hypoxia. Nat. Biomed. Eng. 1, 0057 (2017).

11. Liu, Z. et al. A reversible fluorescent probe for real-time quantitative monitoring of cellular glutathione. Angew. Chem. Int. Ed. 56, 5812-5816 (2017).

12. Erbas-Cakmak, S. et al. Molecular logic gates: the past, present and future. Chem. Soc. Rev. 47, 2228-2248 (2018).

13. Long, L. et al. A mitochondria-specific fluorescent probe for visualizing endogenous hydrogen cyanide fluctuations in neurons. J. Am. Chem. Soc. 140 , 1870-1875 (2018)

14. Jiang, X. et al. Quantitative real-time imaging of glutathione. Nat. Commun. 8 , 16087 (2017).

15. Umezawa, K., Yoshida, M., Kamiya, M., Yamasoba, T. \& Urano, Y. Rational design of reversible fluorescent probes for live-cell imaging and quantification of fast glutathione dynamics. Nat. Chem. 9, 279-286 (2017).

16. Yu, Q. et al. Semisynthetic sensor proteins enable metabolic assays at the point of care. Science 361, 1122-1126 (2018).

17. Berney, C. \& Danuser, G. FRET or no FRET: a quantitative comparison. Biophys. J. 84, 3992-4010 (2003).

18. Broussard, J. A., Rappaz, B., Webb, D. J. \& Brown, C. M. Fluorescence resonance energy transfer microscopy as demonstrated by measuring the activation of the serine/threonine kinase Akt. Nat. Protoc. 8, 265-281 (2013).

19. Jares-Erijman, E. A. \& Jovin, T. M. FRET imaging. Nat. Biotechnol. 21, 1387-1395 (2003).

20. Gu, Y. et al. Ratiometric detection of mitochondrial thiol with a two-photon active AIEgen. ACS Appl. Bio Mater. 2, 3120-3127 (2019).

21. Zhang, Y. et al. Near-infrared hybrid rhodol dyes with spiropyran switches for sensitive ratiometric sensing of ph changes in mitochondria and drosophila melanogaster first-instar larvae. ACS Appl. Bio Mater. 2, 4986-4997 (2019).

22. Chen, M. et al. Malonitrile-functionalized tetraphenylpyrazine: aggregationinduced emission, ratiometric detection of hydrogen sulfide, and mechanochromism. Adv. Funct. Mater. 28, 1704689 (2018).

23. McMahon, B. K. \& Gunnlaugsson, T. Selective detection of the reduced form of glutathione (GSH) over the oxidized (GSSG) form using a combination of glutathione reductase and a $\mathrm{Tb}$ (III)-cyclen maleimide based lanthanide luminescent 'switch on' assay. J. Am. Chem. Soc. 134, 10725-10728 (2012).

24. Haidekker, M. A. \& Theodorakis, E. A. Ratiometric mechanosensitive fluorescent dyes: design and applications. J. Mater. Chem. C. 4, 2707-2718 (2016).

25. Zhang, G., Palmer, G. M., Dewhirst, M. W. \& Fraser, C. L. A dual-emissivematerials design concept enables tumour hypoxia imaging. Nat. Mater. 8, 747-751 (2009).

26. Kasha, M. Characterization of electronic transitions in complex molecules Disc. Faraday Soc. 9, 14 (1950).

27. Guo, J. et al. Mechanical insights into aggregation-induced delayed fluorescence materials with anti-kasha behavior. Adv. Sci. 6, 1801629 (2019)

28. Demchenko, A. P., Tomin, V. I. \& Chou, P. T. Breaking the kasha rule for more efficient photochemistry. Chem. Rev. 117, 13353-13381 (2017).

29. Qian, H. et al. Suppression of Kasha's rule as a mechanism for fluorescent molecular rotors and aggregation-induced emission. Nat. Chem. 9, 83-87 (2017).

30. Lin, J.-A. et al. Bending-type electron donor-donor-acceptor triad: dual excited-state charge-transfer coupled structural relaxation. Chem. Mater. 31, 5981-5992 (2019)

31. Gong, Y. et al. Multiwavelength anti-kasha's rule emission on self-assembly of azulene-functionalized persulfurated arene. J. Phys. Chem. C 123, 22511-22518 (2019)

32. Chen, C. L., Chen, Y. T., Demchenko, A. P. \& Chou, P. T. Amino proton donors in excited-state intramolecular proton-transfer reactions. Nat. Rev. Chem. 2, 131-143 (2018).

33. Itoh, T. Fluorescence and phosphorescence from higher excited states of organic molecules. Chem. Rev. 112, 4541-4568 (2012).

34. Fu, W. et al. Rational design of near-infrared aggregation-induced-emissionactive probes: in situ mapping of amyloid-beta plaques with ultrasensitivity and high-fidelity. J. Am. Chem. Soc. 141, 3171-3177 (2019).

35. $\mathrm{Wu}, \mathrm{X}$. et al. In vivo and in situ tracking cancer chemotherapy by highly photostable NIR fluorescent theranostic prodrug. J. Am. Chem. Soc. 136, 3579-3588 (2014).

36. Brancato, G. et al. Dual fluorescence through Kasha's rule breaking: an unconventional photomechanism for intracellular probe design. J. Phys. Chem. B 119, 6144-6154 (2015).

37. Sung, J. et al. $\mathrm{S}_{2}$ fluorescence dynamics of meso-aryl-substituted subporphyrins. Angew. Chem. Int. Ed. 52, 12632-12635 (2013).

38. $\mathrm{Li}, \mathrm{X}$. et al. A three-dimensional ratiometric sensing strategy on unimolecular fluorescence-thermally activated delayed fluorescence dual emission. Nat. Commun. 10, 731 (2019). 
39. Jiang, W., Fu, Q., Fan, H., Ho, J. \& Wang, W. A highly selective fluorescent probe for thiophenols. Angew. Chem. Int. Ed. 46, 8445-8448 (2007).

40. Wu, D., Chen, L., Kwon, N. \& Yoon, J. Fluorescent probes containing selenium as a guest or host. Chem. 1, 674-698 (2016).

41. Lee, M. H. et al. Liposomal texaphyrin theranostics for metastatic liver cancer. J. Am. Chem. Soc. 138, 16380-16387 (2016).

42. Zhou, Q. et al. Enzyme-activatable polymer-drug conjugate augments tumour penetration and treatment efficacy. Nat. Nanotechnol. 14, 799-809 (2019).

43. Hu, X. et al. Polyprodrug amphiphiles: hierarchical assemblies for shaperegulated cellular internalization, trafficking, and drug delivery. J. Am. Chem. Soc. 135, 17617-17629 (2013).

44. Jiang, X. et al. Quantitative imaging of glutathione in live cells using a reversible reaction-based ratiometric fluorescent probe. ACS Chem. Biol. 10, 864-874 (2015).

45. Guo, Z. et al. Photocaged prodrug under NIR light-triggering with dualchannel fluorescence: in vivo real-time tracking for precise drug delivery. Sci. China Chem. 61, 1293-1300 (2018).

46. Frisch, M. et al. Gaussian 16 Revision A. 03. 2016. (Gaussian Inc., Wallingford CT, 2016).

47. Zhao, Y. \& Truhlar, D. G. The M06 suite of density functionals for main group thermochemistry, thermochemical kinetics, noncovalent interactions, excited states, and transition elements: two new functionals and systematic testing of four m06-class functionals and 12 other functionals. Theor. Chem. Acc. 120, 215-241 (2007).

48. Marenich, A. V., Cramer, C. J. \& Truhlar, D. G. Universal solvation model based on solute electron density and on a continuum model of the solvent defined by the bulk dielectric constant and atomic surface tensions. J. Phys. Chem. B 113, 6378-6396 (2009).

49. Niu, Y. et al. Molecular materials property prediction package (momap) 1.0: a software package for predicting the luminescent properties and mobility of organic functional materials. Mol. Phys. 116, 1078-1090 (2018).

\section{Acknowledgements}

This work was supported by NSFC/China (21788102, 21636002, 21622602, and 21908060), National Key Research and Development Program (2017YFC0906902 and 2016YFA0200300), Shanghai Municipal Science and Technology Major Project (Grant 2018SHZDZX03), the Innovation Program of Shanghai Municipal Education Commission, Scientific Committee of Shanghai (15XD1501400), Programme of Introducing Talents of Discipline to Universities (B16017), the Shuguang Program (18SG27), the China Postdoctoral Science Foundation (2019M651417), and Singapore University of Technology and Design (SUTD) and the SUTD-MIT International Design Centre (IDC) [T1SRCI17126, IDG31800104]. The authors would like to acknowledge the use of the computing service of SUTD-MIT IDC and National Supercomputing Centre, Singapore.

\section{Author contributions}

All the experiments were conducted by L.S. and C.Y. with the supervision of Z.G. W.Z and H.T. All the computational work was conducted by W.C. with the supervision of X.L. All the femtosecond time-resolved transient absorption spectroscopic experiments were conducted by J.W. with the supervision of W.L. All authors discussed the results and co-wrote the manuscript.

\section{Competing interests}

The authors declare no competing interests.

\section{Additional information}

Supplementary information is available for this paper at https://doi.org/10.1038/s41467 020-14615-3.

Correspondence and requests for materials should be addressed to Z.G., X.L. or W.-H.Z.

Peer review information Nature Communications thanks Daniel Escudero and the other, anonymous, reviewer(s) for their contribution to the peer review of this work.

Reprints and permission information is available at http://www.nature.com/reprints

Publisher's note Springer Nature remains neutral with regard to jurisdictional claims in published maps and institutional affiliations.

(c) (i) Open Access This article is licensed under a Creative Commons Attribution 4.0 International License, which permits use, sharing, adaptation, distribution and reproduction in any medium or format, as long as you give appropriate credit to the original author(s) and the source, provide a link to the Creative Commons license, and indicate if changes were made. The images or other third party material in this article are included in the article's Creative Commons license, unless indicated otherwise in a credit line to the material. If material is not included in the article's Creative Commons license and your intended use is not permitted by statutory regulation or exceeds the permitted use, you will need to obtain permission directly from the copyright holder. To view a copy of this license, visit http://creativecommons.org/ licenses/by/4.0/.

(C) The Author(s) 2020 